\title{
Construction and Performance of Multigap RPC in Streamer and Avalanche mode
}

\author{
S. Narita ${ }^{a}$ Y. Hoshi ${ }^{b}$ K. Neichi ${ }^{c}$ A. Yamaguchi ${ }^{d}$ \\ a Iwate University, Department of Electrical and Electronic Engineering \\ Morioka, Iwate, 020-8551, Japan \\ b Tohoku-Gakuin University, Department of Electronic Engineering \\ Tagajo, Miyagi, 980-8537, Japan \\ c Tohoku-Gakuin University, Department of Business Administration \\ Sendai, Miyagi, 980-8511, Japan \\ d Tohoku University, Department of Physics \\ Sendai, Miyagi, 980-8578, Japan
}

\begin{abstract}
We have constructed the multigap RPC and investigated its performance in counting efficiency and the time response for the cosmic-ray muons. The multigap structure of resistive plate chamber (RPC) is supposed to be helpful to obtain reasonably large signal without degrading the time resolution. We found that an average time resolution for 3 gap RPC in avalanche mode was about $0.48 \mathrm{~ns}$. The time resolution is considered to be inversely proportional to the gap size.
\end{abstract}

Key words: RPC, Multigap, Avalanche, Streamer PACS:

\section{Introduction}

Resistive plate chamber (RPC) has been developed in high energy physics field and it is currently used for various experiments as a low cost tracking detector with good space and timing resolutions $[1-6]$.

There are two modes in operating RPC; avalanche and streamer modes. The signal generated in avalanche mode is not large enough and the amplifier device is usually required in the signal readout system. On the other hand, the streamer mode, which gives large signal, is preferred to use to simplify the front-end electronics. However, it sometimes has a problem in decreasing efficiency for high rate beam. Therefore, these two modes are used properly upon experiment purposes.

Besides the operation mode, the performance of the RPC is characterized by the condition of the gas gap. In the RPC, the gas gap between two parallel plates has two roles; one is for creating the primary ionization clusters, the other is for gas gain. Then, the signal charge from the RPC depends on the gas gap. Making the gap wider is helpful to obtain larger signal especially in operation of avalanche mode. However the time resolution of RPC gets worse with a larger gas gap because the larger path for the avalanche may give a larger fluctuations in the arrival time.

In order to avoid such degradation in time resolution, the multigap structure of RPC was proposed [7-9]. Although its performance have been investigated, the systematic study is still necessary to clarify the characteristics. The multigap RPC consists of resistive plates and gas gaps stacked alternately, and electrodes are placed on the outer surface of the outer most resistive plates. The resistive plates are transparent to the fast signals generated by the avalanches (and streamers) inside each gas gap. The induced signal on the readout pads (strips) becomes the sum of the individual avalanche signal. Therefore, larger signal is expected. In the case of that the internal resistive plates are all electrically floating, the time jitter are reduced by the small size of the subgaps.

In this study, we constructed some types of multigap RPC and tested them systematically to clarify the characteristics for further applications. 


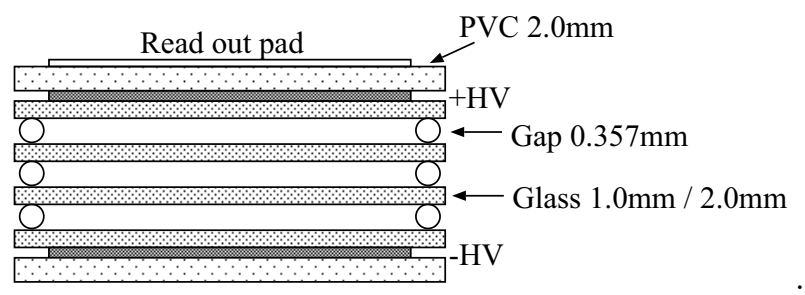

Fig. 1. Multigap RPC (3 gap).

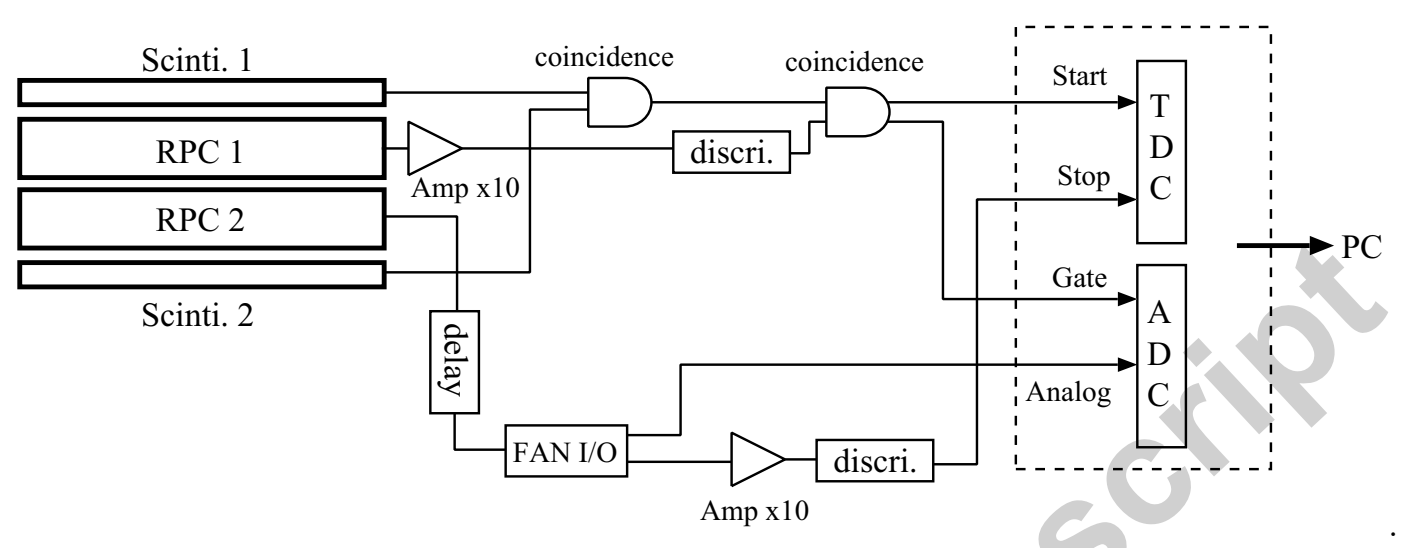

Fig. 2. Experimental setup and readout logic.

\section{Experiment}

We constructed three types of multigap RPC with a variety of the number of gaps and the thickness of resistive plate, which is floating glass with with $10 \times 10 \mathrm{~cm}^{2}$ rectangular shape. The surface and volume resistivity of the glass were $\sim 1 \times 10^{12} \Omega / \square$ and $\sim 6 \times 10^{12} \Omega \cdot \mathrm{cm}$, respectively. A carbon tape was pasted on outer surfaces of both uppermost and lowermost glasses in order to supply the high voltage to the electrodes. The inner electrodes were not connected anywhere and electrically floating. On one of the outer surfaces of the electrodes, the copper readout pad was placed to read out the signals from the RPC. The structures of the multigap RPC were as follows; 3 gap with $1 \mathrm{~mm}$ glasses, 3 gap with $2 \mathrm{~mm}$ glasses, and 5 gap with $1 \mathrm{~mm}$ glasses The cross section of one of the multigap RPCs are shown in Fig. 1 The fishing-strings were placed between the gaps and the gap distance was adjusted to $0.36 \mathrm{~mm}$. The RPC was operated with the gas mixture of $\mathrm{Ar} 50 \%, \mathrm{C}_{4} \mathrm{H}_{10}$ $8 \%, \mathrm{C}_{2} \mathrm{H}_{2} \mathrm{C}_{4} 37 \%$, and $\mathrm{SF}_{6} 5 \%$, and $\mathrm{C}_{4} \mathrm{H}_{10} 8 \%, \mathrm{C}_{2} \mathrm{H}_{2} \mathrm{~F}_{4}$ $87 \%$, and $\mathrm{SF}_{6} 5 \%$. The former mixture was utilized for operating in streamer mode, and the latter one was in avalanche mode.

The performance of the multigap RPCs was investigated using cosmic rays. Fig. 2 shows the experimental setup and the logic of the readout system. Two identical multigap RPCs were placed between scintillation counters. The signal from the one of the multigap RPCs were divided into two with a linear fan-in/out. One signal went into ADC, and the other went into TDC through a discriminator. As seen in Fig.2, cosmic rays were triggered by two scintillation counters and one of the multigap RPSs in our system. In the TDC measurement, the start and the stop timings were determined by two identical RPCs, i.e. the RPC- 1 and the RPC-2 in Fig.2, respectively. In this configuration, the sigma of TDC distribution gives an information of intrinsic time resolution of RPC. The time resolution was extracted as $\sigma / \sqrt{2}$, where $\sigma$ is the standard deviation of the Gaussian function fitted to the TDC distribution.

\section{Results and Discussion}

Fig.3 shows the HV dependencies of the detection efficiency of 3 gap RPC with $1 \mathrm{~mm}$ glasses. The efficiency was comparable to that of single gap RPC. The plateau HV values were obtained from the efficiency curve for each type of RPC.

The ADC distributions for 3 gap RPC with $1 \mathrm{~mm}$ glassed in streamer mode is shown in Fig.4. The peak around 70ch is supposed to correspond to the signals grown by the avalanche process, and the broad peak above $\sim 120 \mathrm{ch}$ corresponds to the streamer ones. Note that the highest peak around $20 \mathrm{ch}$ is considered as the pedestal.

The time resolution of the multigap RPC was derived from the standard deviation of the Gaussian function fitted to the TDC distribution. The time resolutions at the plateau HV region for the constructed RPCs are shown in Fig.5. In this study, the time resolutions for the RPCs were estimated averaging over the values for several $\mathrm{HV}$ values on plateau region. The time resolutions estimated are sum- 


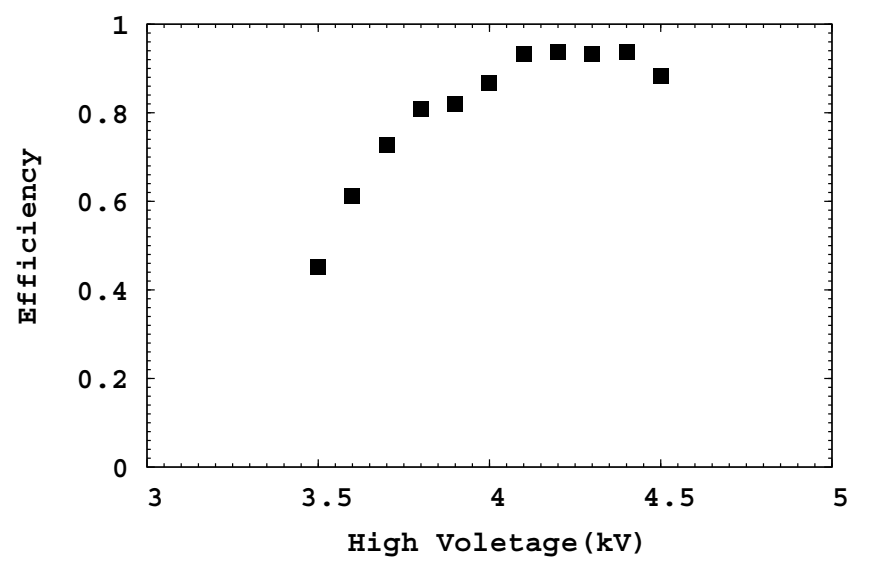

Fig. 3. Efficiency vs. applied high voltage for 3 gap RPC with $1 \mathrm{~mm}$ glasses operated in streamer mode.

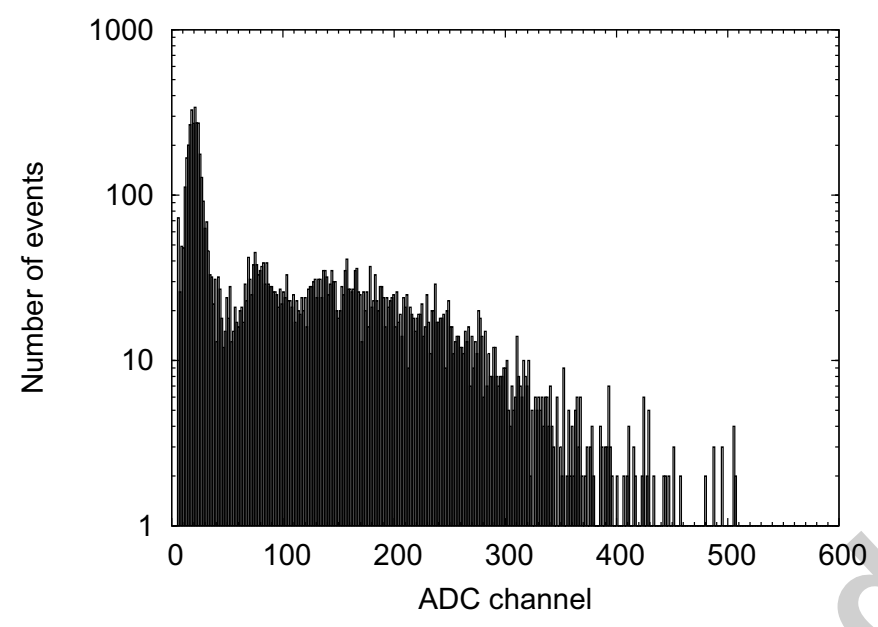

Fig. 4. ADC distribution for 3 gap RPC with $1 \mathrm{~mm}$ glasses in avalanche mode at $\mathrm{HV}$ of $7.0 \mathrm{kV}$.

marized in the Table1. The time jitter in the signal formation in the steamer mode should be larger than that in the avalanche mode, and that is the reason why the time resolution of the streamer mode was worse. In avalanche mode, the time resolution is likely to get better for using the inner glass of $1 \mathrm{~mm}$ thick than $2 \mathrm{~mm}$ thick. Also the dependence on the number of gaps were seen. In the streamer mode, the dependence on the thickness of inner glass and the number of gas gap is smaller than that in the avalanche mode.

\section{Summary}

We have investigated the characteristics of some types of the multigap RPC in both avalanche and streamer modes. In avalanche mode, the time resolution is taken to be inversely proportional to the thickness of inner glass resistive plate. An average time resolution of $0.48 \mathrm{~ns}$ for 3 gap RPC was obtained at the working $\mathrm{HV}$ plateau for cosmic rays. The multigap structure improve the time resolution of the $\mathrm{RPC}$ and it is promising for various applications.
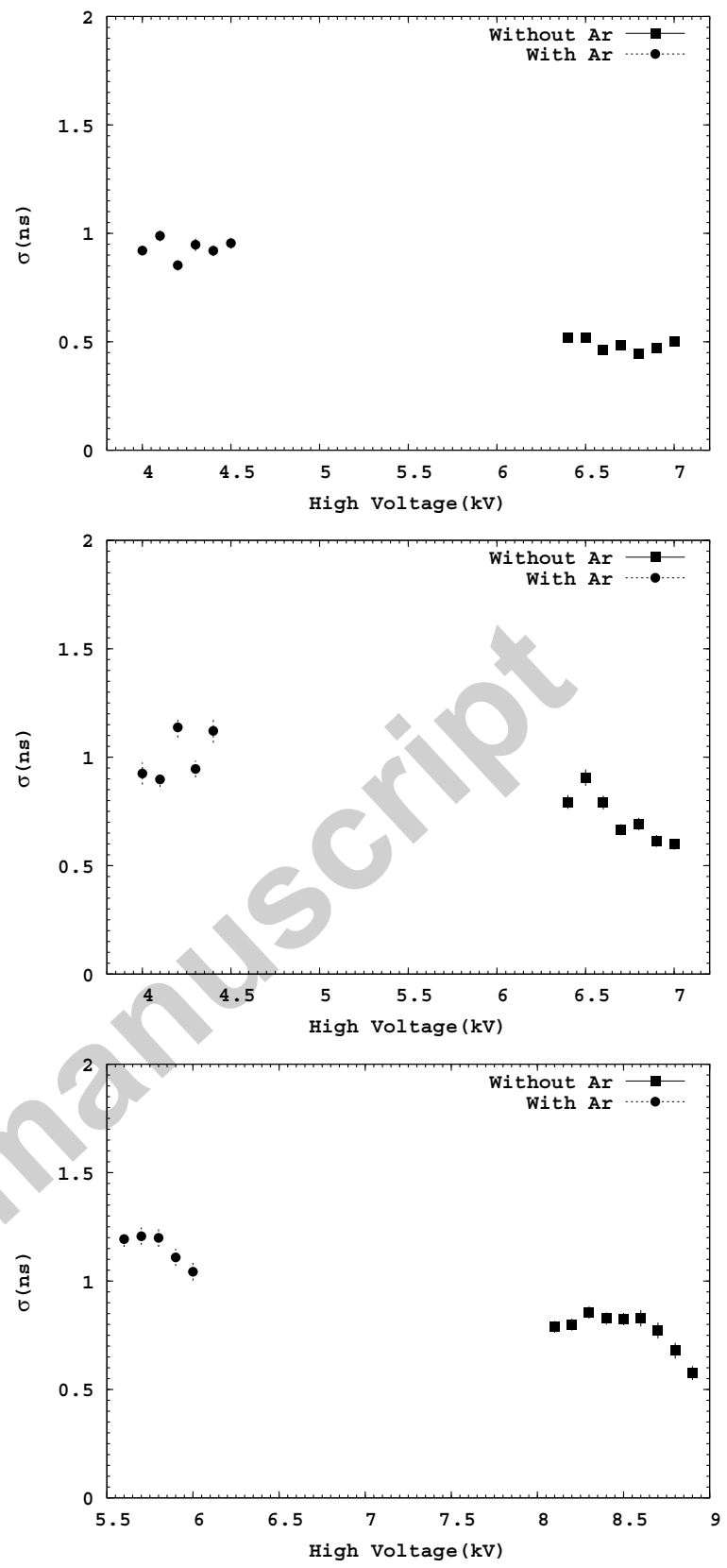

Fig. 5. Time resolutions for the plateau HV in avalanche and streamer mode for 3 gaps with $1 \mathrm{~mm}$ inner glasses (top), 3 gaps with $2 \mathrm{~mm}$ inner glasses (middle), and 5 gaps with $1 \mathrm{~mm}$ inner glasses (bottom).

Table 1

Time resolution of each type of multigap RPC in each operation mode.

\begin{tabular}{|l|c|c|}
\hline & avalanche $[\mathrm{ns}]$ & streamer $[\mathrm{ns}]$ \\
\hline 3 gap $($ glass $=1 \mathrm{~mm})$ & 0.48 & 0.92 \\
\hline 3 gap $($ glass $=2 \mathrm{~mm})$ & 0.71 & 1.00 \\
\hline 5 gap $($ glass $=1 \mathrm{~mm})$ & 0.76 & 1.14 \\
\hline
\end{tabular}




\section{References}

[1] R. Santonico and R. Cardarelli, Nucl. Instr. and Meth. A 187 (1981) 377.

[2] R.Cardarelli and R.Santonico, Nucl. Instr. Meth, A 263 (1988) $20-25$.

[3] A.Abashian, et al., Nucl. Instr. Meth, A 449 (2000) 112.

[4] ATLAS Collaboration, Muon Spectrometer Technical Design Report, CERN/LHCC 97-22.

[5] CMS Collaboration, The Muon Project Technical Design Report, CERN/LHCC 97-32.

[6] ALICE Collaboration, Time of Flight System Technical Design Report, CERN/LHCC 2000-12.

[7] E. Cerron Zaballos et al., Nucl. Instr. and Meth. A 374 (1996) 132.

[8] A. Akindinov et al., Nucl. Instr. and Meth. A 456 (2000) 16.

[9] P. Colrain et al., Nucl. Instr. and Meth. A 456 (2000) 62. 